\title{
Optical emission spectroscopy for diagnosis of diamond growth and etching processes in microwave plasma
}

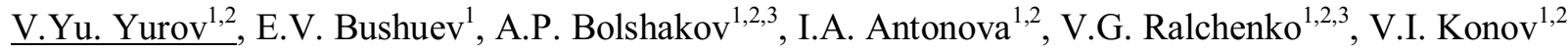 \\ ${ }^{1}$ General Physics Institute RAS, Moscow 119991, Russia, yurov6591@gmail.com \\ ${ }^{2}$ National Research Nuclear University MEPhI, Moscow 115409, Russia \\ ${ }^{3}$ Harbin Institute of Technology, 150001 Harbin, P.R. China
}

For a better control and understanding of growth process of CVD single crystal diamond in microwave (MW) plasma it is important to monitor a large number of the gas phase parameters (chemistry, temperature, concentrations, etc.). A relatively simple and non-perturbing method to measure the plasma parameters is the optical emission spectroscopy (OES) [1, 2]. The OES advantage is also in a fast response to variation of the plasma parameters in the reactor. Here we present the results of OES application to characterize the $\mathrm{MW}$ plasma $(2.45 \mathrm{GHz})$ in course of diamond deposition in $\mathrm{H}_{2}-\mathrm{CH}_{4}$ mixtures and etching in pure $\mathrm{H}_{2}$.

The emission from the plasma passed through a quartz window in the CVD reactor chamber and an image was formed by a lens onto a matted silica plate (OES configuration (I)). The emission collected by a $0.6 \mathrm{~mm}$ diameter quartz fiber was directed to a spectrometer (Ocean Optics HR 4000, wavelength range of 400$800 \mathrm{~nm}$, spectral resolution $0.6 \mathrm{~nm}$. The fiber tip was translated across the image of the plasma to record spatially resolved emission spectra along $\mathrm{X}$ or $\mathrm{Z}$ axes, parallel and perpendicularly to the substrate, respectively, with the istance of -25 to $+25 \mathrm{~mm}$ along the $\mathrm{X}$ axis (counting from the plasma center), and from zero (the substrate position) to $50 \mathrm{~mm}$ along $\mathrm{Z}$ axis. The probed volume was a cylinder with diameter of 1.5-2 mm directed along the plasma ball diameter ( $\mathrm{Y}$ direction), thus the spatial resolution of $\sim 1.5 \mathrm{~mm}$ in the OES was obtained both for $\mathrm{X}$ and $\mathrm{Z}$ axes [2].

In another OES configuration (II) a M833 spectrometer (Solar Laser System) with high spectral resolution of $0.01 \mathrm{~nm}$, equipped with a double grating with 2400 lines/mm and CCD image sensor Hamamatsu S104201006, was used. The optical fiber system collected the emission from a cylinder of $12 \mathrm{~mm}$ diameter directed horizontally along the plasma ball diameter (Y direction). The gas temperature was evaluated from OES data measured in the central region of plasma by the analysis of the $(0,0)$ band of $\mathrm{C}_{2}$ Swan system $\left(\mathrm{d}^{3} \Pi_{\mathrm{g}} \rightarrow \mathrm{a}^{3} \Pi_{\mathrm{u}}\right)$ following the procedure described in $[3,4]$. A thermal equilibrium between heavy species' translational and rotational modes, and a Boltzmann distribution of the rotational levels of the considered vibrational state are assumed.

The spectroscopy of the Swan transitions of the $C_{2}$ molecule $(\lambda=516 \mathrm{~nm})$ with well-resolved rotational components was used to measure the gas temperature with accuracy of $\pm 150{ }^{\circ} \mathrm{C}$ upon wide variation, $1 \div 13 \%$, in methane concentration [5]. The rotational temperature $T_{\text {rot }}$ only weakly depends on the absorbed MW power (Fig.1). It increase from $\sim 3000 \mathrm{~K}$ at low $(2 \%) \mathrm{CH}_{4}$ content to $4000-4500 \mathrm{~K}$ at higher $(7-13 \%) \mathrm{CH}_{4}$ concentrations.

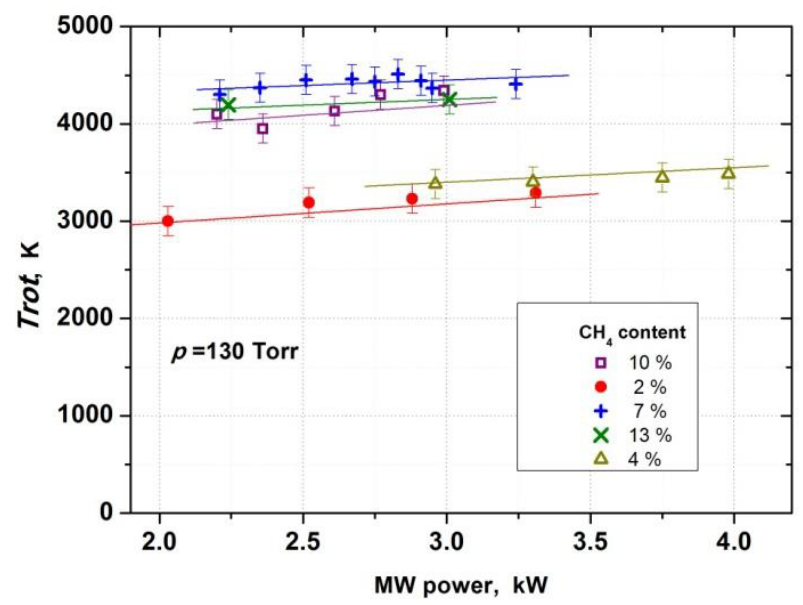

Fig. 1. Rotational temperature $T_{\text {rot }}$ in the core of plasma cloud measured from $\mathrm{C}_{2}$ fine structure spectrum vs $\mathrm{MW}$ power at $\mathrm{CH}_{4}$-consentations: $2,7,410,13,10 \%$, and pressure $p=130$ Torr

The EOS data are useful for estimation of MW power density in the discharge. As the atomic hydrogen line $\mathrm{H}_{\alpha}$ is excited mainly by electron impact, the intensity of $\mathrm{H}_{\alpha}$ line gives an information about the plasma size [6]. With spatially resolved EOS for atomic hydrogen line $\mathrm{H}_{\alpha}$ the intensity profiles of plasma glow were reconstructed in different cross sections. This, in turn, allows correct assessment of the MW power density, which eventually governs the rates of all chemical reactions in the plasma $[1,7]$.

We used two different approaches to measure $\mathrm{H}_{\alpha}$ profiles in vertical $(\mathrm{Z})$ and horizontal $(\mathrm{X})$ directions of the discharge. The first one was based on spatially-resolved EOS in configuration (I) and spectrometer measured $\mathrm{H}_{\alpha}$ line intensity. The second method implies using photographs of the plasma captured with a digital mirror camera Canon EOS 650D through a red $\mathrm{H}_{\alpha}-$ filter passing only the $\mathrm{H}_{\alpha}$ line emission $(\lambda=656.5 \mathrm{~nm} \pm 6 \mathrm{~nm})$. The spatial profiles of the $\mathrm{H}_{\alpha}$ line emission along $\mathrm{X}$ and $\mathrm{Z}$ axes were determined from those images, and the shape of the plasma was very well fitted with Gaussian profiles, with further calculation of the volume and microwave power density (MWPD) values. It was important to save images as a RAW-data, that keep linearity in intensity in the photo [1]. The both approaches gave consisted results and very good fits with Gaussian profiles.

Diamond etching with atomic hydrogen in a microwave plasma is an important process in preparation of hydrophobic surfaces, highly stable interfaces modified with a variety of biomolecules, in pre-treatment of single crystal (SC) substrates in a $\mathrm{H}_{2}$ or $\mathrm{H}_{2}-\mathrm{O}_{2}$ mixtures to eliminate defects before homoepitaxy. Due to low etching 
rates the etching kinetics measurements may take much time, and these data still are rather rare.

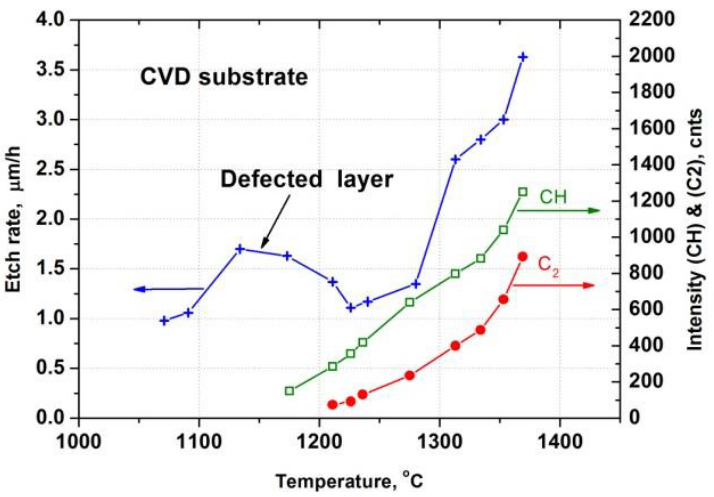

Fig. 2. Etching rate for CVD sample (crosses) in pure $\mathrm{H}_{2}$ plas$\mathrm{ma}$ and intensities of $\mathrm{CH}$ (open squares) and $\mathrm{C}_{2}$ (filed circles) lines in OES spectra of the plasma vs substrate temperature at pressure 130 Torr

The EOS is also interesting for study diamond etching in pure hydrogen, which is relevant for substrate pretreatment in diamond growth technology. Since the etching of diamond supplies carbon species into the plasma, OES is able to monitor the emission lines from $\mathrm{CH}$ and $\mathrm{C}_{2}$ radicals. High resolution OES configuration (II) was used in such experiments. Simultaneously, the etching rate of (001) plane of SC diamond was measured with a low coherent interferometry [7]. The etch rate increases with the substrate temperature $T_{\mathrm{s}}$, however, nonmonotonically (Fig. 2). The etching rate spike in the temperature range of $1170-1230{ }^{\circ} \mathrm{C}$ is caused by enhanced etch of a defective surface layer formed by the sample polishing. At higher $T_{\mathrm{s}}$ the etching rate follows Arrhenius law with activation energy of $43 \pm 5 \mathrm{Kcal} / \mathrm{mol}$.

At the lower $T_{\mathrm{s}}$ of $1092{ }^{\circ} \mathrm{C}$ only the hydrogen lines $\mathrm{H}_{\beta}$ and $\mathrm{H}_{\gamma}$ are seen in OES spectra, but with the temperature increase to $T_{\mathrm{s}}=1369{ }^{\circ} \mathrm{C}$ the $\mathrm{CH}$ line at $431 \mathrm{~nm}$ and the $\mathrm{C}_{2}$ peak $(516 \mathrm{~nm})$ are revealed, as the supply of carbon to the plasma becomes significant. While the primary etching products of CVD sample are hydrocarbons $\mathrm{C}_{\mathrm{x}} \mathrm{H}_{\mathrm{y}}$, the $\mathrm{CH}$ and dimer $\mathrm{C}_{2}$ radicals observed in OES, form by further reactions in the plasma.

Both $\mathrm{I}_{\mathrm{CH}}$ and $\mathrm{I}_{\mathrm{C} 2}$ intensities monotonically increase with etch temperature, following in general the enhanced etch rate. The $\mathrm{CH}_{\mathrm{x}}$ radicals are assumed to be primary etching products, which are then consumed in a part for conversion to a number of carbon-containing species, such as $\mathrm{C}_{2} \mathrm{H}_{2}, \mathrm{CH}_{4}, \mathrm{C}_{2}$, and others to reach an equilibrium in the plasma [8]. We checked that $\mathrm{CH}$ and $\mathrm{C}_{2}$ lines appeared in OES spectra only in presence of a hot diamond substrate in the reactor. No lines associated with carboncontaining species were observed in the plasma optical emission spectra in absence of the substrates under identical MW power and pressure. Therefore the $\mathrm{CH}$ and $\mathrm{C}_{2}$ emission we monitored was surely not due to the plasma etching of carbon contamination from the reactor chamber walls.

As the substrate temperature in the present work was tuned by variation of microwave power $P$, rather than by an independent heating/cooling, a question arises if the plasma chemistry (concentration of atomic $\mathrm{H}$ ) contributes to the etching kinetics in addition to the main effect of the substrate temperature. The elementary processes and concentration of species in the plasma are determined by the microwave power density (MWPD) $P / V$, where $V$ is the plasma volume. We checked how the MWPD depends on the absorbed power, using spatial profiling of $\mathrm{H}_{\alpha}$ line intensity, and we found the MWPD to be almost constant. This suggests a stability of plasma chemistry and atomic hydrogen flux towards the diamond surface, therefore we assign the observed etch effects solely to the temperaturedepended surface chemical reactions.

The measurement of OES intensities of $\mathrm{CH}$ and $\mathrm{C}_{2}$ lines could be a simple and convenient qualitative indicator of etching rate of diamond in $\mathrm{H}_{2}$ plasma, and presumably also in plasmas with more complex compositions, such as $\mathrm{H}_{2}-\mathrm{O}_{2}$ or $\mathrm{H}_{2}-\mathrm{Ar}$.

This work was supported by the Russian Science Foundation, grant No. 14-12-01403-P.

\section{References}

1. M.A. Lobaev, S.A. Bogdanov, et al. Method of power density determination in microwave discharge, sustained in hydrogen-methane gas mixture // Diam. Relat. Mater. 2016. V. 66. P. 177-182.

2. A.P. Bolshakov, V.G. Ralchenko, et al. High-rate growth of single crystal diamond in microwave plasma in $\mathrm{CH} 4 / \mathrm{H} 2$ and $\mathrm{CH} 4 / \mathrm{H} 2 / \mathrm{Ar}$ gas mixtures in presence of intensive soot formation // Diam. Relat. Mater. 2016. V.62. P. 49-57.

3. X. Duten, A. Rousseau, et al. Rotational temperature measurements of excited and ground states of $\mathrm{C}_{2}\left(\mathrm{~d}^{3} \Pi g-\mathrm{a}^{3} \Pi u\right)$ transition in a $\mathrm{H}_{2} / \mathrm{CH}_{4} 915 \mathrm{MHz}$ microwave pulsed plasma // J. Appl. Phys. 1999. V. 86. P. 5299-5301.

4. G. Lombardi, F. Bénédic, et al. Determination of gas temperature and $\mathrm{C}_{2}$ absolute density in $\mathrm{Ar} / \mathrm{H} 2 / \mathrm{CH} 4$ microwave discharges used for nanocrystalline diamond deposition from the $\mathrm{C}_{2}$ Mulliken system.// Plasma Sources Sci. Technol. 2004. V. 13. P. 375-386.

5. E.V. Bushuev, V.Yu. Yurov, et al. Express in situ measurement of epitaxial CVD diamond film growth kinetics // Diam. Relat. Mater. 2017. V. 72. P. 61-70.

6. J. Ma, M.N.R. Ashfold, and Y. A. Mankelevich, Validating optical emission spectroscopy as a diagnostic of microwave activated $\mathrm{CH} 4 / \mathrm{Ar} / \mathrm{H} 2$ plasmas used for diamond chemical vapor deposition // J. Appl. Phys. 2009. V.105. P. 043302.

7. E.V. Bushuev, V.Yu. Yurov, et al. Synthesis of single crystal diamond by microwave plasma assisted chemical vapor deposition with in situ low-coherence interferometric control of growth rate // Diam. Relat. Mater. 2016. V. 66. P. 83-89.

8. J. Ma, J.C. Richley, et al. Probing the plasma chemistry in a microwave reactor used for diamond chemical vapor deposition by cavity ring down spectroscopy //J. Appl. Phys. 2008. V. 104. P. 103305. 